\title{
Strees Analysis in Elastic Half Space Due To a Thermoelastic Strain
}

\author{
Ayaz Ahmad \\ Department of Mathematics NIT Patna Bihar India 800005
}

\begin{abstract}
The stress distribution on elastic space due to nuclei of thermo elastic strain distributed uniformly on the circumference of a circle of radius $R$ situated in the place $z=\lambda$ of the elastic semi space of Hookean model has been discussed by Nowacki: The Force stress and couple stress have been determined. The fore stress reduces to the one obtained by Nowacki for classical elasticity.
\end{abstract}

\section{I.}

\section{Introduction:}

Analysis of stress distribution in elastic space due to nuclei of thermoelastic strain distributed uniformly on the circumference of a circle of radius $r$ situated in the plane $\mathrm{Z}=\mathrm{h}$ of the elastic semi space of Hookean model has been discussed by Nowacki.

This note is an extension of the analysis of above problem for micropolar elastic semi-space. Force stress ${ }^{\sigma} \mathrm{ji}$ and couple stress ${ }^{\mu} \mathrm{ji}$ have been determined due to presence of nuclei of thermoelastic strain situated in the place $\mathrm{Z}=\mathrm{h}$ inside the semi space. The force stress reduces to the one obtained by Nowascki for classical elasticity.

\section{Basic Equations:}

We consider a homogenous isotropic elastic material occupying the sami infinite region $\mathrm{Z} \geq \mathrm{O}$ in cylindrical polar coordinate system $(\mathrm{r}, \theta, \mathrm{Z})$. It has been shown by Nowacki [64] that is in the case when the 2 macrodisplacement vector $\frac{\rightarrow}{u}$ and microrotation $\frac{\rightarrow}{w}$ depend only on $\mathrm{r}$ and $\mathrm{z}$ the basic equations of equilibrium of micro-polar theory of elasticity are decomposed into two mutually independent sets. Here we shall be concerned with the set $\frac{\overrightarrow{ }}{u}=\left(\mathrm{u}_{\mathrm{r}}, \mathrm{O}, \mathrm{u}_{\mathrm{z}}\right)$ and the rotation vector $\frac{\vec{w}}{w}=\left(\mathrm{O}, \phi_{\theta}, \mathrm{O}\right)$ :

$$
\begin{aligned}
& (\mu+\alpha)\left(\nabla^{2}-\frac{1}{{ }_{r}}\right) u_{r}+(\lambda+\mu-\alpha) \frac{\partial e}{\partial r}-2 \alpha \frac{\partial \phi_{\theta}}{\partial z}=\varsigma \frac{\partial T}{\partial r} \\
& (\mu+\alpha)\left(\nabla_{u_{z}}^{2}-+(\lambda+\mu-\alpha) \frac{\partial e}{\partial r}+2 \alpha \cdot \frac{1}{r} \frac{\partial}{\partial r}\left(r \phi_{\theta}\right)=\varsigma \frac{\partial T}{\partial z}\right. \\
& (\gamma+\in)\left(\nabla^{2}-\frac{1}{r} \phi_{\theta}+2 \alpha\left(\frac{\partial u_{r}}{\partial z}-\frac{\partial u_{z}}{\partial r}\right)-4 \alpha \phi_{\theta}=0\right. \\
& \text { Where } \quad \text { e } \quad=\quad \frac{1}{r} \frac{\partial}{\partial r}\left(r \mu_{r}\right)+\frac{\partial u_{z}}{\partial z} \\
& \nabla^{2} \quad \equiv \quad \partial_{r}^{2}+\frac{1}{r} \partial_{r}+\partial_{z}^{2} \\
& \zeta \quad=(3 \lambda+2 \mu)^{\alpha} \mathrm{t} \\
& \mathrm{u}_{\mathrm{r}}, \mathrm{u}_{\mathrm{z}}=\text { displacement components } \\
& \phi_{\theta} \quad=\quad \text { Component of rotation vector } \\
& \lambda, \mu, \alpha, \gamma, \in=\text { elastic constants } \\
& \mathrm{T}(\mathrm{r}, \mathrm{z})=\quad \text { temperature distribution } \\
& \alpha_{t}=\text { coefficient of thermal expansion. }
\end{aligned}
$$


To the displacement vector $\frac{\rightarrow}{u}\left(\mathrm{u}_{\mathrm{r}}, \mathrm{O}, \mathrm{u}_{\mathrm{z}}\right)$ and the rotation vector $\frac{\rightarrow}{w}=\left(\mathrm{O}, \phi_{\theta}, \mathrm{O}\right)$ is ascribed the following state of force stress ${ }^{\sigma} \mathrm{ij}$ and couple stress ${ }^{\mu} \mathrm{ij}$

$\sigma_{\mathrm{ij}}=\left\|\begin{array}{lll}{ }^{\sigma} \mathrm{rr} & 0 & { }^{\sigma} \mathrm{rz} \\ 0 & \sigma & 0 \\ \sigma_{\mathrm{zr}} & 0 & { }^{\sigma} \mathrm{zz} \\ \mu_{\mathrm{ij}} & =\|{ }^{\mu} \mathrm{r} \theta & 0 \\ 0 & 0 & { }^{\mu} \theta \mathrm{z} \\ { }^{\mu} \theta \mathrm{r} & { }^{\mu} \mathrm{z} \theta & 0 \\ 0 & & \end{array}\right\|$

\section{Stress-Strain relations :}

The relation between stress tensor $\sigma_{\mathrm{ij}}, \mu_{\mathrm{ij}}$ and displacement $\frac{\rightarrow}{u}$ and rotation $\frac{\rightarrow}{w}$ in the cylindrical coordinates are given by 3

$$
\begin{aligned}
& \sigma_{\text {гг }}=2 \mu \frac{\partial u_{r}}{\partial r}+\lambda e-T \\
& \sigma_{\theta \theta} \quad=2 \mu \frac{u_{r}}{r}+\lambda e-T \\
& \sigma_{z z} \quad=2 \mu \frac{\partial u_{z}}{\partial z}+\lambda e-T \\
& \sigma_{\text {гz }}=\mu\left(\frac{\partial u_{z z}}{\partial r}+\frac{\partial u_{r}}{\partial z}\right)-\alpha\left(\frac{\partial u_{r}}{\partial z}+\frac{\partial u_{z}}{\partial r}\right)+2 \alpha \phi_{\theta} \\
& \sigma_{\text {zr }}=\mu\left(\frac{\partial u_{z}}{\partial r}+\frac{\partial u_{r}}{\partial z}\right)+\alpha\left(\frac{\partial u_{r}}{\partial z}-\frac{\partial u_{z}}{\partial r}\right)-2 \alpha \phi_{\theta} \\
& \mu_{\mathrm{r} \theta} \quad=\quad \gamma\left(\frac{\partial \phi_{\theta}}{\partial r}-\frac{\phi_{\theta}}{r}\right)+\in\left(\frac{\partial \phi_{\theta}}{\partial r}+\frac{\phi_{\theta}}{r}\right) \\
& \mu_{\theta \mathrm{r}} \quad=\gamma\left(\frac{\partial \phi_{\theta}}{\partial r}-\frac{\phi_{\theta}}{r}\right)+\in\left(\frac{\partial \phi_{\theta}}{\partial r}+\frac{\phi_{\theta}}{r}\right) \\
& \mu_{\theta z} \quad=\quad(\gamma-\epsilon) \frac{\partial \phi_{\theta}}{\partial z}, \\
& \mu_{z \theta} \quad=\quad(\gamma-\epsilon) \frac{\partial \phi_{\theta}}{\partial z}
\end{aligned}
$$

4

Following Nowacki [108], we introduce displacement potentials $\phi, \Psi$ and rotation potential V such that

$$
\begin{aligned}
\mu_{\mathrm{r}} & =\frac{\partial \phi}{\partial r}+\frac{\partial^{2} \psi}{\partial{ }_{r} \partial z} \\
\mu_{z}= & \frac{\partial \phi}{\partial z}-\frac{1}{r} \frac{\partial}{\partial r}\left(r \frac{\partial \psi}{\partial r}\right)
\end{aligned}
$$


$\phi_{\theta}=\frac{\partial v}{\partial r}$

Substituting ( 6.3) in (6.2) we get

$$
\begin{aligned}
& (\lambda+2 \mu) \frac{\partial}{\partial r}\left(\nabla^{2} \theta\right)+\frac{\partial^{2}}{\partial z \partial r}\left[(\mu+\alpha) \nabla^{2} \psi-2 \alpha v\right] \mid=\varsigma \frac{\partial T}{\partial r} \ldots \ldots(6.4) \\
& (\lambda+2 \mu) \frac{\partial}{\partial r}\left(\nabla^{2} \theta\right)-\left(\nabla^{2}-\right) \frac{\partial^{2}}{\partial z^{2}}\left[(\mu+\alpha) \nabla^{2} \psi-2 \alpha v\right]=\varsigma \frac{\partial T}{\partial r} \\
& \frac{\partial}{\partial r}\left[(\gamma+\epsilon) \nabla^{2}-4 \alpha\right] v+2 \alpha=\frac{\partial}{\partial r} \nabla 2 \Psi=0
\end{aligned}
$$

The above equations are satisfied if

$$
\begin{aligned}
& \nabla^{2} \nabla^{2} \phi=\mathrm{m} \nabla^{2} \mathrm{~T} \\
& \nabla^{2}\left({ }^{2} \nabla^{2}-1\right) \mathrm{V}=0
\end{aligned}
$$

Where $£^{2}=\frac{(\mu+\alpha) \gamma+\epsilon)}{4 \alpha \mu}, \mathrm{m}=\frac{\varsigma}{\lambda+2 \mu}$,

related by

$$
\nabla^{2} \Psi=-2\left[\left(\frac{\gamma+\epsilon}{4 \alpha}\right) \nabla^{2}-1\right]
$$

To solve (6.5) we write

$$
\begin{aligned}
& \phi \quad=\quad \phi^{\prime} \quad+\quad \phi^{\prime} \\
& \text {..... (6.7) } \\
& \mathrm{V}=\mathrm{V}^{\prime}+\mathrm{V}^{\prime}
\end{aligned}
$$

Where $\phi '$ and V' are particular integrals for non-homogeneous part and $\phi ", \mathrm{~V}$ " are general solutions of homogeneous part. Now for particular integral we have

$\begin{array}{llll}\nabla^{2} & \phi & = & \mathrm{mT} \\ \text { and } & \mathrm{V} & = & 0\end{array}$

and for general solution we have

$$
\begin{array}{llll}
\nabla^{2} & \nabla^{2} & \phi ” & = \\
\nabla^{2}\left(\ell^{2} \nabla^{2}-1\right) & V^{\prime \prime} & = & 0
\end{array}
$$

IV. Solution of the title problem :

We consider nuclei of thermo elastic strain distributed uniformly on the circumference of a circle of radius $\mathrm{r}$ and situated in the plane $\mathrm{z}=\mathrm{h}$ inside the elastic half space. The stress distribution $\sigma_{\mathrm{ij}}$ can be considered as sum of two stress systems $|\bar{S}|$ and $|\bar{S}|$. The system $|\bar{S}|$ constitute stress distribution $\sigma$ 'ij of infinite elastic space containing two nuclei of thermoelastic strains situated in the planes $\mathrm{z}=\mathrm{h}$ and $\mathrm{z}=-\mathrm{h}$ distributed uniformly along the circumferences of the circles, each of radius $r$. The second system $|\overline{\overline{\mid}}|$ constitutes stress distribution $\sigma_{\mathrm{ij}}$ corresponding to elastic semi-space in the isothermal state. The stress $\sigma^{\prime \prime}{ }_{\mathrm{ij}}$ is so chosen that the boundary conditions on the plane $\mathrm{z}=\mathrm{O}$.

are satisfied.

$$
\sigma_{\mathrm{zz}} \quad=0, \quad \sigma_{\mathrm{zr}}=0, \quad \mu_{\mathrm{z} \theta}=0
$$

The thermoelastic displacement potential $\phi^{\prime}$ corresponding to $\sigma^{\prime}$ ij satisfies the equation 7

$\nabla^{2} \phi \quad=\quad \mathrm{m} \delta\left(\mathrm{R}^{\mathrm{r}}-\mathrm{R}\right)[\delta(\mathrm{z}-\mathrm{h})-\delta(\mathrm{z}+\mathrm{h})]$

Where $\mathrm{r}^{2}=\mathrm{x}^{2}+\mathrm{y}^{2}$ and $\delta(\mathrm{x})$ represents Dirac - delta function.

Representing the right hand side of the equations (6.10) by the Fourier Integral 
$\mathrm{m} \delta(\mathrm{r}-\mathrm{R})[\delta(\mathrm{z}-\mathrm{h})-\delta(\mathrm{z}+\mathrm{h}]$
$=\frac{m R}{\pi} \int_{0}^{\infty} \int_{0}^{\infty} \xi J_{o}(\xi r) J_{o}(\xi R)[\operatorname{Cosr}(z-h)-\operatorname{Cosr}(z+h] d \xi d r$

The solution of (6.10) is represented by the integral

$$
\begin{aligned}
& \phi^{\prime}=-\frac{m R}{2} \int_{o}^{\infty} J_{o}(\xi R) J_{o}(\xi r)\left[e^{-} \xi(z-h)-_{e}^{-} \xi(z+h)\right] d . \\
& \xi,|z|-h>0 \\
& =-\frac{m R}{2} \int_{o}^{\infty} J_{o}(\xi R) J_{o}(\xi r)\left[e^{\left.-\xi(z-h)-{ }_{e}^{-} \xi(z+h)\right] d \xi,|z|-h \leq 0}\right.
\end{aligned}
$$

8

The stress distribution for the system $(\bar{S})$ is obtained

$$
\begin{aligned}
\sigma_{\mathrm{rr}}^{\prime} & =2 \mu\left[\left(\frac{\partial^{2} \phi^{\prime}}{\partial r^{2}}\right)-\nabla^{2} \phi^{\prime}\right] \\
& =\quad \operatorname{m\mu R} \int_{o}^{\infty} \xi^{2} J_{o}(\xi R)\left[J_{o}(\xi R)+\frac{1}{\xi r} J_{1}(\xi r)\right]\left[e^{(\xi(z-h)}-e^{-\xi(z+h)}\right] d \xi \\
\sigma \theta \theta^{\prime} & =2 \mu\left(\frac{1}{r} \frac{\partial \phi^{\prime}}{\partial r}-\nabla^{2} \phi^{\prime}\right)=-2 \mu\left(\frac{\partial^{2} \phi^{\prime}}{\partial r^{2}}+\frac{\partial^{2} \phi^{\prime}}{\partial z^{2}}\right) \\
& =\operatorname{m\mu R} \int_{o}^{\infty} \xi^{2} J_{o}(\xi R)\left[J_{o}(\xi r)+J_{o}^{\prime \prime}(\xi r)\right]\left[e^{(\xi(z-h)}-e^{-\xi(z+h)}\right] d \xi
\end{aligned}
$$

V. General Solution for Homogeneous Equations:

Applying Kankel transform to equation (6.9), the general solution for half space is given by

$$
\phi \quad=\int_{o}^{\infty} \xi(A+B \xi z) e^{-\xi z} J o(\xi r) d \xi
$$

and $\quad \mathrm{V}^{\prime}, \quad=\int_{o}^{\infty} \xi\left(L_{e}^{-\xi z}+M e^{-\sigma z}\right) J_{o}(\xi r) d \xi$

where $\sigma^{2}=\xi^{2}+\frac{1}{\ell^{2}}$ and $\mathrm{L}, \mathrm{M}, \mathrm{A}, \mathrm{B}$ are
Equations (6.4) give
\[ \mathrm{L}=-\frac{\lambda+2 \mu}{\mu} \xi \mathrm{B} . \]

Knowing the functions $\phi ", \Psi "$ and V" the force stresses and couple stresses are calculated by the relations $\sigma_{\mathrm{rr}} \quad=2 \mu \quad \frac{\partial u_{r}}{\partial r}+\lambda e=2 \mu \frac{\partial^{2}}{\partial r^{2}}\left(\phi^{\prime \prime}+\frac{\partial \psi^{\prime \prime}}{\partial z}\right)+\lambda \nabla^{2} \phi^{\prime \prime}$ 


$$
\begin{aligned}
& \sigma_{\theta \theta} \quad=2 \mu \quad \frac{1}{r} \frac{\partial}{\partial r}\left(\phi^{\prime \prime}+\frac{\partial \psi^{\prime \prime}}{\partial z}\right)+\lambda \nabla^{2} \phi^{\prime \prime} \\
& \sigma_{\mathrm{zz}}^{\prime} \quad=\quad 2 \mu \quad \frac{\partial}{\partial z}\left[\frac{\partial \phi^{\prime \prime}}{\partial z}-\left(\nabla^{2}-\frac{\partial^{2}}{\partial z^{2}}\right) \psi^{\prime \prime}\right]+\lambda \nabla^{2} \phi^{\prime \prime} \\
& \sigma_{\mathrm{zr}} \quad=\frac{\partial}{\partial r}\left[\mu\left\{2 \frac{\partial \phi^{\prime \prime}}{\partial z}-\left(\nabla^{2}-2 \frac{\partial^{2} z}{\partial z^{2}}\right) \psi^{\prime \prime}\right\}\right. \\
& \left.+\alpha \nabla^{2} \psi^{\prime \prime}-2 \alpha V^{\prime \prime}\right] \\
& \mu{ }_{\mathrm{r} \theta} \quad=\quad(\gamma+\in) \frac{\partial^{2} V^{\prime \prime}}{\partial_{r}{ }^{2}}-(\gamma-\epsilon) \frac{1}{r} \frac{\partial V^{\prime \prime}}{\partial_{r}} \\
& \mu ”_{\theta \mathrm{r}} \quad=\quad(\gamma-\epsilon) \frac{\partial^{2} V^{\prime \prime}}{\partial_{r}{ }^{2}}-(\gamma+\epsilon) \frac{1}{r} \frac{\partial V^{\prime \prime}}{\partial_{r}} \\
& \mu_{z \theta}^{\prime \prime} \quad=\quad(\gamma+\in) \frac{\partial^{2} V^{\prime \prime}}{\partial r \partial z} \\
& \mu_{\theta z} \quad=\quad(\gamma-\epsilon) \frac{\partial^{2} V^{\prime \prime}}{\partial r \partial z}
\end{aligned}
$$

Since the bounding surface $\mathrm{z}=0$ is free from tractions, we have on $\mathrm{z}=\mathrm{O}, \quad|\mathrm{S}|+|\stackrel{\bar{S}}{\mathrm{~S}}|=\quad \mathrm{O}$ Thus

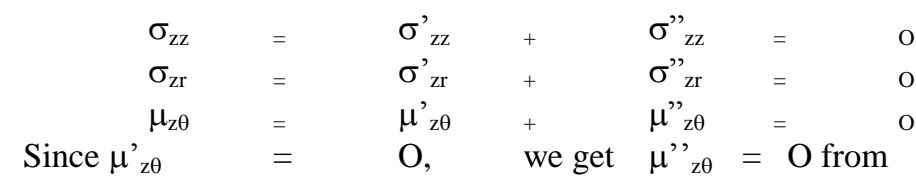

This gives

$$
\mathrm{L} \quad=\quad-\quad \mathrm{M} \frac{\sigma}{\xi}
$$

$$
\mathrm{L} \quad=--\left(\frac{\lambda+2 \mu}{\mu}\right) \xi \mathrm{B}
$$

Also, from (6.16) we get

$$
\mathrm{M} \quad=\quad-\mathrm{L} \frac{\xi}{\sigma}=\left(\frac{\lambda+2 \mu}{\mu}\right)\left(\frac{\xi^{2}}{\sigma}\right) \mathrm{B}
$$

The solution of equation

Is obtained as

$$
\nabla^{2} \psi^{\prime \prime}=-\frac{1}{2 \alpha}\left[(\gamma+\epsilon) \nabla^{2}-4 \alpha\right] V^{\prime \prime}
$$

$$
\psi^{\prime \prime}=\frac{\lambda+\mu}{\mu} \int_{o}^{\infty} B\left(\frac{\lambda+2 \mu}{\lambda+\mu} \xi z e^{-\xi z}+2 a_{o} \frac{\xi 3}{\sigma} e_{-}^{-\sigma z}\right) J_{o}(\xi r) d \xi
$$

Where $\mathrm{a}_{\mathrm{o}} \quad \frac{(\lambda+\in)(\lambda+2 \mu}{4 \mu(\lambda+\mu)}$ 
Boundary conditions (6.18) 1, 2 yield

$$
\begin{aligned}
& \mathrm{A}=4 \text { ao } \quad \xi^{2} \mathrm{P}(\xi) \\
& \mathrm{B}=\frac{(2 \mu}{(\lambda+\mu)} \mathrm{P}(\xi)
\end{aligned}
$$

Where $\mathrm{P}(\xi) \quad=\frac{m R \xi J_{o}(\xi R) e^{-\xi h}}{1+2 a_{o} \xi 2(1-\xi / \sigma)}$

Substituting expressions for $\phi$ ", $\Psi$ " and V" with values of A and B in (6.20), we obtain $\sigma{ }^{\prime}{ }_{\mathrm{ij}}$ and $\mu$ ' ${ }_{\mathrm{ij}}$ with the help of the relations

$$
\begin{aligned}
& \sigma_{\mathrm{zz}}=3 \mu \int_{o}^{\infty}\left[4 a_{0} \xi^{2}-\frac{2 \mu}{\lambda+\mu}(2-\xi z] P(\xi) \xi^{3} e-{ }^{-\xi z} J_{o}(\xi r) d \xi\right. \\
& +2 \mu \int_{o}^{\infty}\left[\left(1+\frac{\mu}{\lambda+\mu}\right)(1-\xi z) e^{-\xi z}-2 a_{o} \xi^{2} e^{-\sigma z}\right] \xi^{3} P(\xi) J_{o}(\xi r) d \xi \\
& -\frac{4 \mu}{\lambda+\mu} \int_{o}^{\infty} \xi^{3} e^{-\xi z} p(\xi) J_{o}(\xi r) d \xi \\
& \sigma_{\mathrm{zr}} \quad=2 \mu \int_{o}^{\infty}\left[\frac{2 \mu}{\lambda+\mu}(1-\xi z)-4 a_{o} \xi^{2}\right] P(\xi) e^{-\xi z} J_{o}{ }^{\prime}(\xi r) d \xi \\
& +4(\mu-\alpha) \int_{o}^{\infty}\left[1+\frac{\mu}{\lambda+\mu} e^{-\xi z}+a_{o} \xi^{3}(1 / \sigma-\sigma) e^{-\sigma z}\right] P(\xi) \xi^{3} J_{o}^{\prime}(\xi r) d(\xi) \\
& +4 \mu \int_{o}^{\infty}\left[1+\frac{\mu}{\lambda+\mu}(\xi z-2) e^{-\xi z}+2 a_{o} \xi \sigma e^{-\sigma z}\right] \xi^{3} P(\xi) J_{o}^{\prime}(\xi r) d \xi \\
& +\frac{4 \alpha}{\lambda+\mu} \int_{o}^{(\lambda+2 \mu} \xi^{3}\left(e^{-\xi z}-\frac{\xi}{\sigma} e^{-\sigma z}\right) P(\xi) J_{o}^{\prime}(\xi r) d \xi
\end{aligned}
$$

$$
\begin{aligned}
& \mu_{r \theta}^{\prime \prime}=\frac{-2(\lambda+2 \mu)}{\lambda+\mu} \int_{o}^{\infty}\left(e^{-\xi z}-\frac{\xi}{\sigma} e^{-\sigma z}\right)\left[(\gamma+\epsilon) J_{o}^{\prime \prime}(\xi r)-(\gamma-\epsilon) \cdot \frac{1}{r} J_{o}^{\prime}(\xi r)\right] x \xi^{3} P(\xi) \\
& \mu_{z \theta}^{\prime \prime}=\frac{2(\gamma+\epsilon)(\lambda+2 \epsilon)}{\lambda+\mu} \int_{o}^{\infty}\left(e^{-\xi z}-e^{-\sigma z}\right) \xi^{4} P(\xi) J_{o}^{\prime}(\xi r) d \xi
\end{aligned}
$$

Stress distribution in the elastic half space is obtained by adding (6.13) and (6.21)

Thus

$$
\begin{aligned}
& \sigma_{r r}=\sigma_{r r}^{\prime}+\sigma_{r}{ }^{\prime \prime} \\
& =m \mu R \int_{o}^{\infty}\left[J_{o}(\xi r)+\frac{1}{\xi r} J_{1}(\xi r)\right]\left[e^{\xi(z-h)}-e^{-\xi(z-h)}\right] \xi^{2} J_{o}(\xi R) d \xi \\
& +2 \mu \int_{o}^{\infty}\left[4 a_{o} \xi^{2}+\frac{2 \mu}{\lambda+\mu} \xi z P\right]\left[\frac{1}{\xi r} J_{1}(\xi r)-J_{o}(\xi r)\right] \xi^{3} P(\xi) e^{-\xi z} d \xi \\
& +4 \mu \int_{o}^{\infty}\left[\left(1+\frac{\mu}{\lambda+\mu}\right)(1-\xi z) e^{-\xi z}-2 a_{o} \xi^{2} e^{-\sigma z}\right]\left[\frac{1}{\xi r} J_{1}(\xi r)-J_{o}(\xi r)\right] \xi^{3} P(\xi) d \xi \\
& -\frac{4 \mu \lambda}{\lambda+\mu} \int_{o}^{\infty} \xi^{3} e^{-\xi z} p(\xi) j_{0}(\xi r) d \xi
\end{aligned}
$$




$$
\sigma_{\theta \theta}=\sigma_{\theta \theta}{ }^{\prime}+\sigma_{\theta \theta}{ }^{\prime \prime}
$$

$$
\begin{aligned}
& =m \mu R \int_{o}^{\infty}\left[e^{\xi(z-h)}-e^{-\xi(z+h)}\right] \frac{1}{\xi r} J_{1}(\xi r) J_{o}(\xi R) d \xi \\
& +2 \mu \int_{o}^{\infty}\left[4 a_{o} \xi^{2}+\frac{2 \mu}{\lambda+\mu} \xi z\right] \frac{\xi^{2}}{r} P(\xi) e^{-\xi z} J_{1}(\xi r) d \xi \\
& --4 \mu \int_{o}^{\infty}\left[\left(1+\frac{\mu}{\lambda+\mu}\right)(1-\xi z) e^{-\xi z}-2 a o \xi^{2} e^{-\sigma z}\right] \frac{\xi 2}{r} P(\xi) J_{1}(\xi r) d \xi \\
& -\frac{4 \lambda \mu}{\lambda+\mu} \int_{o}^{\infty} \xi^{3} e^{-\xi z} P(\xi) J_{o}(\xi r) d \xi \\
& \sigma_{z z}=\sigma_{z z}^{\prime}+\sigma_{z z}{ }^{\prime \prime} \\
& =-m \mu R \int_{o}^{\infty}\left[e^{-\xi(z-h)}-e^{-\xi(z+h)}\right] \xi^{2} J_{o}(\xi R) J_{1}(\xi r) d \xi \\
& +2 \mu \int_{o}^{\infty}\left[4 a_{o} \xi^{2}-\frac{2 \mu}{\lambda+\mu}(2-\xi z)\right] \xi^{3} e^{-\xi z} P(\xi) J_{o}(\xi r) d \xi \\
& +2 \mu \int_{o}^{\infty}\left[\left(1+\frac{\mu}{\lambda+\mu}\right)(1-\xi z) e^{-\xi z}-2 a_{o} \xi^{2} e^{-\sigma z}\right] \xi^{3} p(\xi) J_{o}(\xi r) d \xi \\
& -\frac{4 \lambda \mu}{\lambda+\mu} \int_{o}^{\infty} \xi^{3} e^{-\xi z} P(\xi) J_{o}(\xi r) d \xi \\
& \sigma_{z r}=\sigma_{z r}+\sigma_{z r} "
\end{aligned}
$$$$
=-m \mu R \int_{o}^{\infty} \xi^{2}\left[e^{\xi(z-h)}-e^{-\xi(z+h)}\right] J_{o}(\xi R) J_{1}(\xi r) d \xi
$$$$
-2 \mu \int_{o}^{\infty}\left[\frac{2 \mu}{\lambda+\mu}(1-\xi z)-4 a_{o} \xi^{2}\right] \xi^{3} P(\xi) e^{-\xi z} J_{1}(\xi r) d \xi
$$$$
-4(\mu-\alpha) \int_{o}^{\infty}\left[\left(1+\frac{\mu}{\lambda+\mu}\right) e^{-\xi z}+a_{o}\left(\frac{1}{\sigma}-\sigma\right) \xi^{3} e^{-\sigma z}\right] \xi^{3} P(\xi) J_{1}(\xi r) d \xi
$$$$
-4 \mu \int_{o}^{\infty}\left[\left(1+\frac{\mu}{\lambda+\mu}\right)(\xi z-2) e^{-\xi z}+2 a_{o} \xi \sigma e^{-\sigma z}\right] \xi^{3} P(\xi) J_{1}(\xi r) d \xi
$$$$
-\frac{4 \alpha(\lambda+2 \mu)}{\lambda+\mu} \int_{o}^{\infty}\left[\left(e^{-\xi z}-\frac{\xi}{\sigma} e^{-\sigma z}\right) \xi^{3} P(\xi) J_{1}(\xi r) d \xi\right]
$$$$
\mu_{r \theta}=\mu_{r \theta}^{\prime \prime}=-\frac{2(\lambda+2 \mu)}{\lambda+\mu} \int_{0}^{\infty}\left[\left(e^{-\xi z}-\frac{\xi}{\sigma} e^{-\sigma z}\right)\right]\left[\xi r J_{2}(\xi r)-\in \xi J_{o}(\xi r)\right] x P(\xi) d \xi
$$$$
\mu_{z \theta}=\mu_{z \theta} "=\frac{-2(\gamma+\epsilon)(\lambda+2 \mu)}{\lambda+\mu} \int_{0}^{\infty}\left(e^{-\xi z}-e^{-\sigma z}\right) \xi^{4} P(\xi) J_{1}(\xi r) d \xi
$$ 


$$
\begin{aligned}
& \sigma_{r r}-\sigma_{\theta \theta}=\left(\sigma_{r r}^{\prime}+\sigma_{r r}^{\prime \prime}\right)-\left(\sigma_{\theta \theta}^{\prime}+\sigma_{\theta \theta}^{\prime \prime}\right) \\
& =\left(\sigma_{r r}^{\prime}-\sigma_{\theta \theta}^{\prime}\right)+\left(\sigma_{r r}^{\prime \prime}-\sigma_{\theta \theta}^{\prime \prime}\right) \\
& =-m \mu R \int_{o}^{\infty}\left[e^{\xi(z-h)}-e^{-\xi(z+h)}\right] \xi^{2} J_{o}(\xi R) J_{2}(\xi r) d \xi
\end{aligned}
$$

$+2 \mu \mathrm{R} \int_{o}^{\infty}\left[\left(4 a_{o} \xi^{2}+\frac{2 \mu}{\lambda+\mu} \xi z\right) e^{-\xi z}+2\left(1+\frac{\mu}{\lambda+\mu}\right)(1-\xi z) e^{-\xi z}-2 a_{o} \xi^{2} e^{-\sigma z}\right] p(\xi)$

$$
x J_{2}(\xi r) d \xi \ldots
$$

For $\alpha=\mathrm{O}$, the micropolar couple stress vanishes and in that case $\gamma=\epsilon=\mathrm{O}, \mathrm{a}_{0}=\mathrm{O}, \sigma=0$. Thus we get from (6.22)

$$
\begin{aligned}
& \sigma_{r r}=m u R \int_{o}^{\infty}\left[J_{o}(\xi r)+\frac{1}{\xi r} j_{1}(\xi r)\right]\left[e^{\xi(z-h)}-e^{-\xi(z+h)}\right] J_{o}(\xi r) d \xi \\
& +\frac{2 \mu}{1-2 v} \int_{0}^{\infty} P(\xi) e^{-\xi z}\left[(2-\xi z) J_{o}(\xi r)+(2 v-2+\xi z) \frac{J_{1}(\xi r)}{\xi r}\right] \xi^{3} d \xi \\
& \sigma_{\theta \theta}=m u R \int_{o}^{\infty}\left[J_{o}(\xi r)+\frac{1}{\xi r} J_{1}(\xi r)\right]\left[e^{\xi(z-h)}-e^{-\xi(z+h)}\right] \xi^{2} J_{o}(\xi R) d \xi \\
& +\frac{2 \mu}{1-2 v} \int_{\circ}^{\infty}\left[\left(2 v J_{o}(\xi r)-(2 v-2 \xi z) \frac{J_{1}(\xi r)}{\xi r}\right] P(\xi) e^{-\xi z} \xi^{3} d \xi\right. \\
& \sigma_{z z}=-m u R \int_{o}^{\infty}\left[e^{\xi(z-h)}-e^{-\xi(z+h)}\right] \xi^{2} J_{o}(\xi R) J_{1}(\xi r) d \xi \\
& 17 \\
& +\frac{2 \mu}{1-2 v} \int_{0}^{\infty} P(\xi) \xi^{4} e^{-\xi z} J_{o}(\xi r) d \xi \\
& \sigma_{\mathrm{zr}}=\sigma_{\mathrm{rz}} \quad=-m u R \int_{o}^{\infty}\left[e^{\xi(z-h)}-e^{-\xi(z+h)}\right] \xi^{2} J_{o}(\xi R) J_{1}(\xi r) d \xi \\
& -\frac{2 \mu}{1-2 v} \int_{0}^{\infty}(1-\xi z) P(\xi) \xi^{3} e^{-\xi z} J_{1}(\xi r) d \xi \\
& \operatorname{ur} \theta=\mathrm{u} \theta \mathrm{r}=0 \\
& \text { where } \mathrm{P}(\xi) \text { reduces to (1-2v) } e^{-h} \mathrm{~J}_{\mathrm{o}}(\xi \mathrm{R}) \text {. } \\
& \text { Results in }(6,23) \text { have been obtained in for Hookean thermo elasticity. }
\end{aligned}
$$




\section{References}

[1]. Palov, N.A. Fundamental equations of the theory of asymmetric elasticity (in Russian). Prikil. Mat. Mekh. 28 (1964). 401.

[2]. Peterson, M.E., Rev. Geophys. 11, 355, 1973.

[3]. Paria, G., Advances in Applied Mechanics, Vol. 10, p. 73, Academic Press, New York (1967)

[4]. Puri, P. Int. J. Engng. Sci. 11, 735 (1973).

[5]. Paria, G.and Wilson, Proc. Camb. Phil. Soc. 58, 527 (1972)

[6]. Papadepolos, M. (1963) : The elastodynamics of moving loads, Jt. Australian Maths. Soc. 3., 79-92.

[7]. Robin, P.Y.F., AMER, Mineral. 59, 1286, 1974.

[8]. Rayleigh Lord, Proc. Lond. Math. Soc. 20, 225 (1888).

[9]. Roy Choudhuri and Loknath Debnath, Magneto thermo-elasticity plane waves in Rotating Media, Int. J. Engg. Sci. Vol. 21, No. 2, pp. 155-169, (1983).

[10]. Schaefer, H. : Versuch einer Elastizitatetheorie des sweidimensionalen Cosserat - Kontinuum, Miss. Angew. Math. Festschrift Tollmien, Berlin, 1962, Akademia Verlag.

[11]. Now ack. W.: Couple- Stresses in the Theory of Thermo-elasticity. Proc. Of the IUTPM symposis Vienna June $22-28$ 1966. Springer- verlag vier 1968.

[12]. Nay feh. A. and Nemat Nasser. J. Appl. Mech 39, Trans. ASME. 94, Ser. E, 108 (1972)

[13]. Purc. P. Int. J. Engng. Sci- 11.735 (1973).

[14]. Robin Py. F, AMER, MINERAL, 59, 1286, 1974.

[15]. Roy Choudhuri and Loknath Deba nath, Magneto Thermo- elasticity plane waves in Rotating Media, int. J- Engg. Sci. Vol., 21. No. 2 PP 155-169 (1983).

[16]. Wilkenson J.P.D. J. accounst. Soc. Am 49, 2002

[17]. Erigen A.C. Proc $9^{\text {th }}$ Midwestern Mechanics cong. Part I wiley 2006. 